\title{
Resultados auditivos y hallazgos quirúrgicos en pacientes con cirugía bilateral por otoesclerosis
}

\author{
Audiological outcome and surgical findings in patients with bilateral surgery \\ for otosclerosis
}

Paul H. Délano $\mathbf{R}^{1,2}$, Andrés Alvo $\mathbf{V}^{1}$, Alejandro 0jeda $\mathbf{S}^{1}$, Carlos Stott $\mathrm{C}^{1}$.

\section{RESUMEN}

Introducción: A pesar de las ventajas de la audición biaural, el manejo quirúrgico del segundo oído en pacientes con otoesclerosis bilateral fue por años un tema controversial debido a su riesgo potencial de hipoacusia sensorioneural.

objetivo: Describir los resultados audiológicos y quirúrgicos en pacientes operados bilateralmente por otoesclerosis en nuestro hospital entre 1988-2011.

Material y método: Estudio retrospectivo de 56 pacientes en quienes se realizó cirugía de otoesclerosis en ambos oídos, mediante revisión de fichas clínicas.

Resultados: En 13,5\% de 415 pacientes se realizó cirugía bilateral. La edad promedio fue 44,2 \pm 7,7 (hombres) y 42,0 $\pm 10,4$ años (mujeres). La mejoría de los umbrales auditivos aéreos promedios en la primera y segunda cirugía fue de 33,6 y 29,2 dB respectivamente, mientras que la de los umbrales óseos fue de 7,0 y 3,0 dB correspondientemente. Las dificultades quirúrgicas para la primera y segunda cirugía fueron, respectivamente: platina flotante $0 \%$ y $2,5 \%$, platina obliterada $5,4 \%$ y $5,1 \%$, nicho profundo $5,4 \%$ y $0 \%$, facial proscidente $38,5 \%$ y $43,2 \%$, facial dehiscente $18,2 \%$ y $13,8 \%$ y sección de cuerda del tímpano $2 \%$ y $5,7 \%$.

Discusión: Los resultados auditivos del segundo oído son comparables a los de la primera cirugía. Esto, junto a la ausencia de complicaciones significativas, apoya la realización de intervenciones quirúrgicas bilaterales en otoesclerosis.

Conclusión: La cirugía del segundo oído en hipoacusia bilateral por otoesclerosis es una alternativa segura y confiable.

Palabras clave: Otoesclerosis, hipoacusia, audición, cirugía de oído, estapedostomía, estapedectomía.

\begin{abstract}
Introduction: Despite the advantages of binaural hearing, surgical management of the second ear in patients with bilateral otosclerosis was for years a controversial topic due to the risk of sensorineural hearing loss.

Aim: To describe the surgical and audiological results of patients with bilateral surgeries for otosclerosis in our hospital between 1988-2011.

Material and method: Retrospective study based on clinical charts revision.
\end{abstract}

${ }^{1}$ Médico Servicio de Otorrinolaringología, Hospital Clínico Universidad de Chile.

${ }^{2}$ Programa de Fisiología y Biofísica, ICBM, Facultad de Medicina, Universidad de Chile. 
Results: $13.5 \%$ of the 415 patients underwent bilateral surgery. The average age was $44.2 \pm 7.7$ (men) and $42.0 \pm 10.4$ years (women). There was a $33.6 \mathrm{~dB}$ improvement in average air-conduction hearing thresholds in the first surgery and $29.2 \mathrm{~dB}$ for the second, while the mean bone-conduction improved 7.0 and $3.0 \mathrm{~dB}$ for the first and second surgery correspondingly. The surgical difficulties found in the first and second surgeries were, respectively: floating platens $0 \%$ and $2.5 \%$, obliterated platens $5.4 \%$ and $5.1 \%$, deep niche $5.4 \%$ and $0 \%$, facial overhang $38.5 \%$ and $43.2 \%$, dehiscent facial $18.2 \%$ and $13.8 \%$ and chorda tympani section $2 \%$ and $5.7 \%$.

Discussion: Hearing results for the second ear were comparable to the first surgery. This, together with the absence of significant complications, supports a bilateral surgical approach.

Conclusion: Surgery of the second ear in bilateral hypoacusia due to otosclerosis has proven to be a safe and reliable alternative.

Key words: Otosclerosis, hearing loss, auditory, ear surgery, stapedostomy, stapedectomy.

\section{INTRODUCCIÓN}

La otoesclerosis es una enfermedad de causa desconocida, con una base genética de transmisión autosómica dominante de baja penetrancia y expresividad variable ${ }^{1}$. Afecta al hueso de la cápsula ótica provocando hipoacusia en el $0,4 \%$ de la población caucásica. Por otra parte, la presencia de focos otoescleróticos en la histología del hueso temporal, puede alcanzar al $12,5 \%$ de la población normal ${ }^{2}$. Se ha encontrado histología anormal bilateral de los huesos temporales en cerca del $70 \%$ a $80 \%$ de los cráneos que presentan la enfermedad ${ }^{3}$.

La estapedectomía se introdujo hace más de 50 años ${ }^{4}, y$ desde esa fecha la técnica ha sufrido múltiples modificaciones. Actualmente la estapedostomía se ha convertido en la técnica quirúrgica más utilizada. Los resultados de esta cirugía han sido evaluados en múltiples estudios demostrando una alta eficacia para mejorar la audición, con un bajo índice de complicaciones y un resultado estable a largo plazo ${ }^{5,6}$, con tasas de éxito que bordean el $96 \%$ en manos de cirujanos experimentados ${ }^{7}$. La indicación de una cirugía bilateral en otoesclerosis fue cuestionada por sus resultados y su potencial riesgo de hipoacusia sensorioneural y secuelas vestibulares 8 . Es así como pocos estudios en la literatura publicada han dado reportes de la técnica bilateral, de sus beneficios funcionales y complicaciones.

\section{OBJETIVO}

El objetivo general del presente trabajo, es describir las características epidemiológicas y los resul- tados audiológicos y quirúrgicos de los pacientes operados por otoesclerosis con colocación de prótesis bilaterales. Además buscamos determinar la proporción de pacientes operados bilateralmente por otoesclerosis, comparar resultados auditivos del primer con el segundo oído y cotejar hallazgos quirúrgicos de la primera con la segunda cirugía.

\section{MATERIAL Y MÉTODO}

Se realizó un estudio retrospectivo de revisión de fichas clínicas entre enero de 1988 y marzo de 2011 que ingresaron a pabellón del Hospital Clínico de la Universidad de Chile con diagnóstico de otoesclerosis. Se incluyeron en el estudio aquellos pacientes mayores de 15 años que fueron operados con colocación de prótesis (estapedostomía y/o estapedectomía) en ambos oídos por otoesclerosis bilateral.

Se analizaron datos de tipo epidemiológicos (sexo, edad, tiempo entre cirugías), audiológicos mediante el estudio audiométrico (umbrales aéreos, y óseos) con promedios tonales puros (PTP) en 500, 1.000 y 2.000 $\mathrm{Hz}$ antes y después de las cirugías. Se describen los hallazgos intraoperatorios y dificultades quirúrgicas del primer y segundo oído y presencia de complicaciones posquirúrgicas.

En el análisis estadístico se utilizó el programa Excel $^{\circledR}$ 2007, de Microsoft ${ }^{\circledR}$ para el cálculo de promedios, desviación estándar y error estándar, prueba de t pareada para datos paramétricos y prueba de Chi-cuadrado para variables no paramétricas, considerando en ambos casos un $p$ $<0,05$ como significativo. Debido al reducido nú- 
mero de dificultades quirúrgicas y a protocolos operatorios incompletos no podemos realizar un estudio de correlación entre los datos obtenidos entre las dos cirugías, por lo que los datos quirúrgicos se presentan sólo con estadística descriptiva.

\section{RESULTADOS}

Se obtuvieron 415 fichas de pacientes operados por otoesclerosis entre los años 1988 y marzo de 2011. De éstos, 359 pacientes han sido operados de un oído y 56 pacientes fueron operados bilateralmente (112 oídos), lo que corresponde al $13,5 \%$ del total.

Los 56 pacientes operados bilateralmente, se dividen en 18 hombres $(32,1 \%)$ y 38 mujeres $(67,9 \%)$, cuyo promedio de edad en la primera cirugía fue 44,2 $\pm 7,7$ años (promedio \pm desviación estándar) y $42,0 \pm 10,4$ años respectivamente (diferencia edad no significativa). El promedio de días entre cirugías fue de $1.459 \pm 1.056$ días (4,0 años) para los hombres y de $1.298 \pm 1.269$ días (3,6 años) en mujeres (diferencia no significativa). Se encontró que el promedio de tiempo entre cirugías antes del año 2000 fue de 2.517 días, mientras que después del año 2000 fue de 885 días (diferencia no significativa).

No hubo diferencias significativas al comparar el lado (izquierdo/derecho) del primer oído operado en varones ( 9 oídos derechos y 9 izquierdos en primera cirugía), por el contrario hubo una diferencia estadísticamente significativa entre el lado del primer oído operado en mujeres (24 oídos izquierdos contra 14 derechos en primera cirugía, $p<0,001$; prueba Chi-cuadrado).

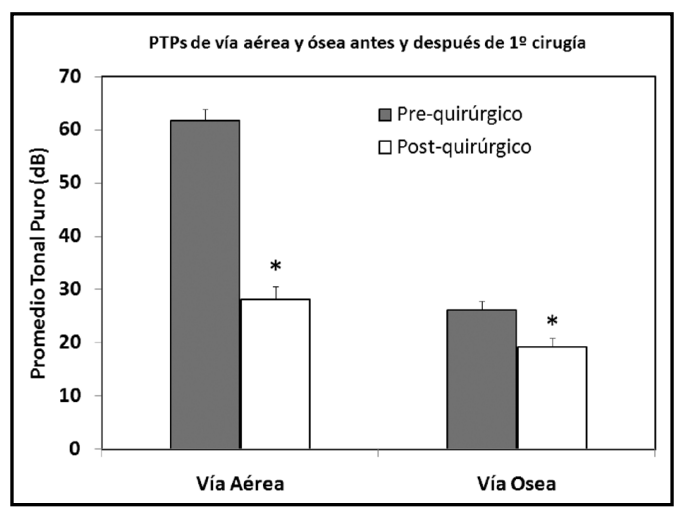

Figura 1. Resultados auditivos posquirúrgicos de la primera cirugía por otoesclerosis bilateral. Los asteriscos representan diferencias de promedio significativas antes y después de cirugía para vía aérea y ósea $(p<0,05)$.

\section{Resultados audiológicos}

De los 56 pacientes se cuenta con datos audiológicos completos en 41 pacientes, por lo que los promedios tonales fueron obtenidos con estos pacientes. EI PTP de la vía aérea antes de la primera cirugía fue de $61,8 \pm 2,1 \mathrm{~dB}$ (promedio \pm error estándar) y el de vía ósea fue de $26,2 \pm 1,7$ $\mathrm{dB}$, mientras que los valores después de la cirugía primaria fueron de $28,2 \pm 2,4 \mathrm{~dB}$ y de 19,2 $\pm 1,7 \mathrm{~dB}$ para vía aérea y ósea respectivamente (Figura 1).

EI PTP de vía aérea promedio antes de la segunda cirugía fue de 59,3 $\pm 2,4 \mathrm{~dB}$ y el de vía ósea de $25,8 \pm$ $1,9 \mathrm{~dB}$, mientras que el valor de los umbrales después de la segunda cirugía fue de $30,1 \pm 2,1$ y 22,8 $\pm 2,6$ para vía aérea y ósea respectivamente (Figura 2).

Al comparar las audiometrías obtenidas después de las dos cirugías, con aquellas realizadas antes de la primera cirugía, la ganancia promedio en umbrales auditivos de vía aérea fue de 29,9 $\pm 3,4 \mathrm{~dB}$ (promedio \pm error estándar) para el oído operado primero y de 30,6 $\pm 3,4 \mathrm{~dB}$ para el oído operado en segundo lugar (diferencia no significativa). En relación a los umbrales de vía ósea, se observó una mejoría de 5,1 $\pm 2,0 \mathrm{~dB}$ en el primer oído, mientras que en la segunda cirugía fue de 7,7 $\pm 2,0 \mathrm{~dB}$ (diferencia no significativa).

Los PTP promedios obtenidos después de las dos cirugías en el oído derecho e izquierdo para vía aérea fueron de $27,5 \pm 2,2 \mathrm{~dB}$ y de 29,5 $\pm 2,7 \mathrm{~dB}$ respectivamente y para vía ósea fueron de $21,7 \pm 2,0$ $\mathrm{dB}$ en oído derecho y de $22,1 \pm 1,8 \mathrm{~dB}$ en el izquierdo (Figura 3). De esta manera, la diferencia obtenida después de las dos cirugías, entre los umbrales de vía

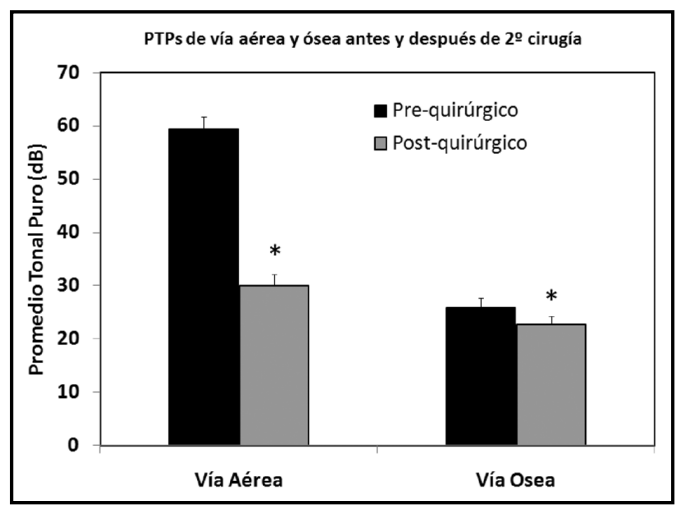

Figura 2. Resultados auditivos posquirúrgicos de la segunda cirugía por otoesclerosis bilateral. Los asteriscos representan diferencias de promedio significativos antes y después de cirugía para vía aérea y ósea $(p<0,05)$. 


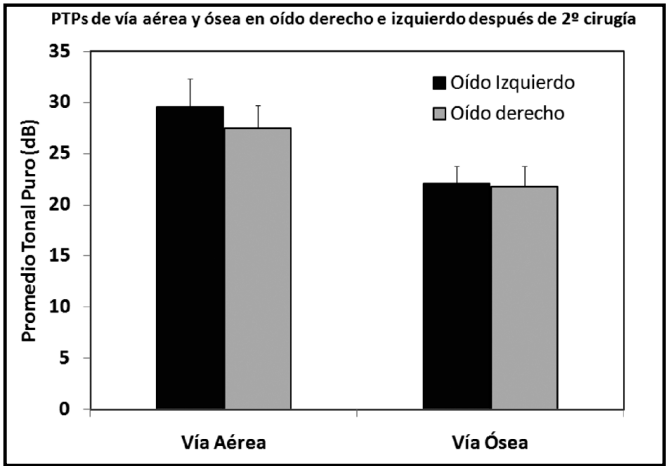

Figura 3. Promedios tonales puros en ambos oídos después de la segunda cirugía con colocación de prótesis por otoesclerosis. Se obtuvieron valores menores a $30 \mathrm{~dB}$ para vía aérea y una diferencia aéreo-ósea menor a $10 \mathrm{~dB}$.

aérea y ósea promedio ("GAP” entre vía aérea y ósea) fueron menores a $10 \mathrm{~dB}$ en ambos oídos $(5,8 \mathrm{~dB}$ en oído derecho y 7,4 dB en oído izquierdo; diferencia estadísticamente no significativa).

\section{Hallazgos quirúrgicos}

En la primera cirugía se describe platina azul en $62,2 \%(\mathrm{~N}=23)$, blanca en $24,3 \%(\mathrm{~N}=9)$, obliterada en $5,4 \%(\mathrm{~N}=2)$ y nicho profundo en $5,4 \%(\mathrm{~N}=2)$. El nervio facial proscidente fue reportado en $38,5 \%$ ( $N=10)$ y dehiscente en $18,2 \%(N=4)$. La sección de la cuerda timpánica alcanzó al 2\% $(\mathrm{N}=1)$.

En la segunda cirugía, se encontró platina azul en $79,5 \%$ ( $N=31$ ), blanca en $12,8 \%(N=5)$, flotante en $2,5 \%(N=1)$ y obliterada en $5,1 \%(N=2)$. La presencia de facial proscidente fue observada en un $43,8 \%$ ( $N=14)$ y dehiscente en $13,8 \%(N=4)$. La sección de cuerda del tímpano descrita alcanzó el 5,7 \% ( $\mathrm{N}=3)$ (Tabla 1).

En cuanto a las prótesis, se reportó utilización de prótesis de Schuknecht ${ }^{\circledR}$ de 4,5 mm de largo y 0,6 mm

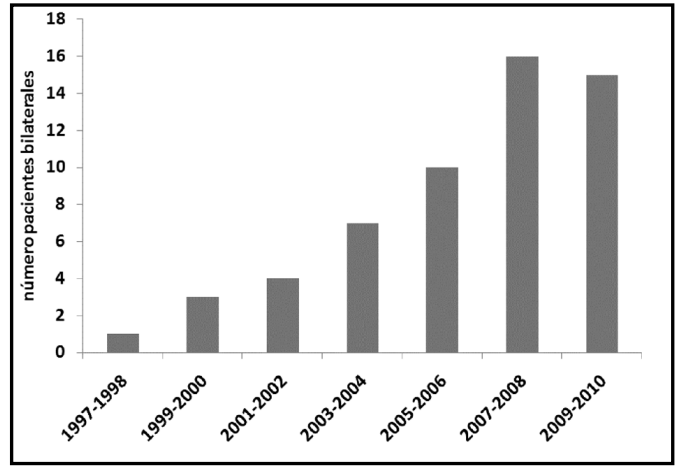

Figura 4. Evolución histórica del número de pacientes operados bilateralmente por otoesclerosis. Se muestra un histograma de frecuencias de pacientes con estapedostomias 0 estapedectomías bilaterales agrupados cada dos años. Se observa un incremento progresivo del número de pacientes operados en los últimos años.

de diámetro en el $86 \%(\mathrm{~N}=43)$ para cirugías de primer oído y $85 \%(\mathrm{~N}=45)$ para las de segundos oídos.

\section{DISCUSIÓN}

El tratamiento quirúrgico de la otoesclerosis a través de estapedectomía o estapedostomía tienen una alta tasa de éxito en la corrección de la hipoacusia conductiva generada por esta enfermedad. Si bien la mayoría de los pacientes con otoesclerosis se presentan en forma bilateral, muchos otólogos aconsejan realizar una cirugía en un solo oído.

La audición biaural es ampliamente superior a la monoaural para tareas psicoacústicas como la localización espacial de sonidos y la extracción de una señal acústica en un ambiente ruidoso9. La localización de una fuente sonora en el espacio se realiza a través de claves biaurales basadas en (i) diferencias temporales interaurales (diferencia de fase entre oídos), y (ii) diferencia de intensidad interaural, provocada por la

Tabla 1. Resultados de hallazgos y dificultades quirúrgicas en cirugías bilaterales por otoesclerosis

\begin{tabular}{|lcc|}
\hline Dificultad quirúrgica & Primer oído & Segundo oído \\
\hline Platina blanca & $24,3 \%(N=9)$ & $12,8 \%(N=5)$ \\
Platina flotante & $0,0 \%(N=0)$ & $2,5 \%(N=1)$ \\
Platina obliterada & $5,4 \%(N=2)$ & $5,1 \%(N=2)$ \\
Nicho profundo & $5,4 \%(N=2)$ & $0,0 \%(N=0)$ \\
Facial proscidente & $38,5 \%(N=10)$ & $43,8 \%(N=14)$ \\
Facial dehiscente & $18,2 \%(N=4)$ & $13,8 \%(N=4)$ \\
Sección de cuerda del tímpano & $2,0 \%(N=1)$ & $5,7 \%(N=3)$ \\
\hline
\end{tabular}


sombra acústica de la cabeza (diferencia de presión sonora entre oídos) $)^{9,10}$. La utilización de los dos oídos permite mejorar la discriminación de palabras en ambiente ruidoso por una mejoría de la relación señal/ruido y por el efecto de sumación de señales auditivas similares. Además, con la biauralidad se ha reportado un claro enriquecimiento en la percepción de la música ${ }^{11}$.

El adecuado manejo quirúrgico de la hipoacusia bilateral por otoesclerosis fue por muchos años un tema controversial, el dilema de operar o no el segundo oído otoesclerótico se basaba en la falta de estudios que respaldaran esta conducta y que evaluaran los beneficios versus los riesgos de este procedimiento. Es así como muchos cirujanos de estapedostomía recomiendan este procedimiento cuando la primera cirugía tuvo éxito con una audición estable y si las indicaciones se mantienen para la cirugía del otro oído ${ }^{12}$.

En nuestro centro esta cirugía fue más frecuentemente efectuada en mujeres, alcanzando al $72,7 \%$ del total, siendo esto concordante con la mayor proporción (2:1) de la enfermedad en el géner $0^{13}$. Si bien, en nuestro hospital se ha incrementado paulatinamente el número de pacientes en que se realiza una cirugía bilateral (Figura 4), éstas representan solo el 13,5\% del total de pacientes operados por otoesclerosis, cifra bastante inferior al $70 \%$ a $80 \%$ de prevalencia de enfermedad bilateral ${ }^{14}$. El intervalo de tiempo encontrado entre la primera y segunda cirugía fue de 1.235 días, sufriendo una reducción importante a partir del año 2000 (2.517 v/ s 885 días). El tiempo óptimo entre cirugías no parece aún claramente establecido en la literatura, la mayoría de los reportes muestran intervalos similares o superiores a nuestra casuística. En nuestro servicio de modo arbitrario, antes de la segunda cirugía se espera un periodo mínimo de un año para observar estabilidad clínica audiológica y ausencia de aparición de complicaciones.

En relación a los hallazgos y dificultades quirúrgicas, la descripción más frecuente de los estribos operados fue de platina azul y como platinas complicadas, destacó la presencia de platinas obliteradas y nichos profundos. También la presencia de facial proscidente y dehiscente se encontró en un elevado porcentaje de los oídos operados. Todos estos hallazgos fueron similares a estudios realizados a nivel local ${ }^{15}$ y publicados en la literatura internacional ${ }^{16}$. La sección de la cuerda del tímpano fue la complicación más frecuentemente reportada, especialmente en la cirugía del segundo oído en donde alcanzó el 5,7\%, sin embargo, esta complicación no siempre produce trastornos del gusto, pero cuando los genera, éstos efectos tienden a ser autolimitados en la totalidad de los pacientes en un periodo máximo de un año $0^{17}$.

Se encontró un significativo éxito posquirúrgico en términos de mejoría de los umbrales auditivos aéreos y óseos, tanto para el primero como en el segundo oído, con recuperación superior o igual a los 29,2 dB de los umbrales aéreos y una mejoría de umbrales óseos igual o superior de $7,7 \mathrm{~dB}$, fenómeno que sería explicado por el reestablecimiento del juego de ventanas. Estos resultados son comparables a un estudio anterior del mismo centro en pacientes operados por otoesclerosis unilateral, que mostró una mejoría de 40 $\mathrm{dB}$ en vía aérea y de $8 \mathrm{~dB}$ en la ósea ${ }^{17}$. Además, en los resultados auditivos no se encontraron diferencias estadísticamente significativas al comparar cirugías de oídos derechos versus izquierdos, conclusión compartida en la literatura publicada ${ }^{18}$. De este modo, un buen resultado en la primera cirugía sin duda que estimula la indicación para un manejo quirúrgico bilateral, pero frente a un resultado discreto o fracaso funcional sigue siendo controvertido proponer un segundo intento.

Frente a la evidencia expuesta relacionada al éxito del manejo quirúrgico bilateral mediante esta cirugía, en términos de mejoría de umbrales auditivos y de calidad de vida, nace la inquietud sobre el tratamiento quirúrgico simultáneo en otoesclerosis. Según reportes, la técnica simultánea -en pacientes seleccionados y realizada por cirujanos experimentados- es un procedimiento seguro que muestra resultados similares a los observados en pacientes operados de forma unilateral, exhibiendo como ventaja una menor tasa de intervenciones médicas para recuperar la biauralidad ${ }^{19}$. En nuestro contexto clínico, el manejo racional de esta patología sigue inclinándose hacia un enfoque conservador, con cirugías secuenciales, realizadas en diferentes tiempos quirúrgicos, con un intervalo de tiempo cercano a un año.

\section{CONCLUSIONES}

Nuestros resultados confirman que la cirugía del segundo oído en hipoacusia bilateral por otoesclerosis es una alternativa segura, confiable y con baja incidencia de complicaciones ${ }^{20,21}$. Los resultados auditivos expuestos en el segundo oído son comparables a los de la primera cirugía, alcanzando muchas veces audición normal bilateral después de la segunda cirugía 22,23 .

La proporción de pacientes con cirugía bilateral es aún muy baja en comparación a la alta prevalencia de 
otoesclerosis clínica bilateral. Además, el tiempo promedio de 3 años entre la primera y segunda cirugía es aún muy extenso, a pesar de que en la última década, este intervalo ha disminuido considerablemente.

\section{BIBLIOGRAFÍA}

1. Causse JR, Causse JB. Otospongiosis as a genetic disease: Early detection, medical management and prevention. Am J Otology 1984; 5: 211-23.

2. Declau F, Van Spaendonck M, Timmermans JP et al. Prevalence of otoesclerosis in an unselected series of temporal bones. Otol Neurotol 2001; 22(5): 596-602.

3. Menger DJ, Tange RA. The aetiology of otoesclerosis: a review of the literature. Clin Otolaryngol Allied Sci 2003; 28(2): 112-20.

4. SHEA JJ. Thirty years of stapes surgery. $J$ Laryngol Otol 1988; 102: 14-9.

5. House hP, Hansen MR, Al Dakhall AA, House JW. Stapedectomy versus stapedotomy: comparison of results with long-term follow-up. Laryngoscope 2002; 112(11): 2046-50.

6. Lanas A, Stott C, Olavaria C. Otosclerosis: Resultados auditivos en estapedectomía y estapedostomía. Rev Otorrinolaringol Cir Cabeza Cuello 2002; 62: 251-4.

7. LIPPY WH. Special problems of otosclerosis surgery. En: Brackmann DE, Shelton C, Arriaga MA, eds, Otologic Surgery. Philadelphia: W.B. Saunders Co., 1994; 347.

8. Shine NP, Rodrigues S, Miller S, Packer P. Bilateral stapedectomy: association between first- and second-ear surgical findings and their effects on the second-ear outcome. Ann Otol Rhinol Laryngol 2008; 117(3): 207-11.

9. McAlpINE D. Creating a sense of auditory space. J Physiol 2005; 566: 21-8.

10. JoHnson BW, Hautus MJ. Processing of binaural spatial information in human auditory cortex: neuromagnetic responses to interaural timing and level differences. Neuropsychologia 2010; 48: 2610-9.
11. Stern RM JR, Colburn HS. Theory of binaural interaction based in auditory-nerve data. IV. A model for subjective lateral position. J Acoust Soc Am 1978; 64(1): 127-40.

12. DanieLs RL, Krieger LW, LipPy WH. The other ear: findings and results in 1,800 bilateral stapedectomies. Otol Neurotol 2001; 22(5): 603-7.

13. GUILD SR. Histologic otosclerosis. Ann Otol 1944; 53: 246-67.

14. Hueb MM, Goycoolea MV, Paparella MM, Oliveira JA. Otosclerosis: the University of Minnesota temporal bone collection. Otolaryngol Head Neck Surg 1991; 105(3): 396-405.

15. Stott C, Royer M, Olmedo R, Martínez C, Valdés C, Ortúzar L. Resultados auditivos de estapedostomías en platinas complicadas. Rev Otorrinolaringol Cir Cabeza Cuello 2006; 66: 89-94.

16. Aedo C, Stott C, Albertz N, Tabilo P. Trastornos del gusto posestapedostomías. Rev Otorrinolaringol Cir Cabeza Cuello 2009; 69: 7-12.

17. Stott C, Nazar R, Manieu D. Manejo quirúrgico en otoesclerosis unilateral. Rev Otorrinolaringol Cir Cabeza Cuello 2005; 65: 111-6.

18. Karkas A, Chahine K, Righini CA, Khirnetkina A, SCHMERBER S. Right versus left stapes surgery: is there a difference? Otol Neurotol 2009; 30: 1067-70.

19. Kujala J, Aalto H, Ramsay H, Hirvonen TP. Simultaneous bilateral stapes surgery. Acta Otolaryngol 2008; 128: 347-51.

20. Albrechet ChG, Fonseca AS, Porto P, Bernardes GC. "Second ear" stapedotomy: is it safe? Acta Otorhinolaryngol Belg 2004; 58: 109-11.

21. De Bruijn AJ, Tange Ra, Dreschler Wa, Grolman W, Schouwenburg PF. Bilateral stapedotomy in patients with otosclerosis: a disability-orientated evaluation of the benefit of second ear surgery. Clin Otolaryngol Allied Sci 1998; 23: 123-7.

22. Kisilevsky VE, Bailie NA, Dutt SN, Halik JJ. Functional results of 394 bilateral stapedotomies evaluated with the Glasgow Benefit Plot. Eur Arch Otorhinolaryngol 2010; 267: 1027-34.

23. De Bruijn AJ, Tange RA, Dreschler WA. Evaluation of second-ear stapedotomy with the Glasgow benefit plot. ORL J Otorhinolaryngol Relat Spec 1999; 61: 92-7. 